\title{
The relation between colour and structural changes in thermochromic systems comprising crystal violet lactone, bisphenol $\mathrm{A}$, and tetradecanol
}

Ondrej Panák (corresponding author)

Department of Graphic Arts and Photophysics, Faculty of Chemical Technology

University of Pardubice, Studentská 95, 53210 Pardubice, Czech Republic

E-mail: ondrej.panak@upce.cz

Tel.: +420466038036

Markéta Držková

Department of Graphic Arts and Photophysics, Faculty of Chemical Technology

University of Pardubice, Studentská 95, 53210 Pardubice, Czech Republic

E-mail: marketa.drzkova@upce.cz

Tel.: +420 466038035

Marie Kaplanová

Department of Graphic Arts and Photophysics, Faculty of Chemical Technology

University of Pardubice, Studentská 95, 53210 Pardubice, Czech Republic

E-mail: marie.kaplanova@gmail.cz

Urban Novak

National Institute of Chemistry, Hajdrihova 19, SI-1001 Ljubljana, Slovenia

E-mail: urban.novak@ki.si

Tel.: +38614760274

Marta Klanjšek Gunde

National Institute of Chemistry, Hajdrihova 19, SI-1001 Ljubljana, Slovenia

E-mail: marta.k.gunde@ki.si

Tel.: +38614760291 


\begin{abstract}
The aim of the paper was to find a direct connection between dynamic colour changes, phase changes and chemical interactions in model three-component leuco dye based thermochromic systems. The model systems, containing crystal violet lactone as a colour former, bisphenol A as a developer and 1tetradecanol as a co-solvent, were analysed by DSC and FTIR spectroscopy and the results were related to the characteristics of the dynamic colour change. The ternary thermochromic systems were also compared with binary mixtures of the co-solvent with the developer and colour former, respectively. The temperatures characterizing the dynamic colour change at decolouration limits were directly related to the solid-liquid transition on heating and liquid-solid transition on cooling, regardless the concentration of bisphenol A. In ternary thermochromic systems, an indistinctive phase transition at the temperatures below the solid-solid (crystal-rotator) transition was observed. The straight connection between the phase transitions and temperatures characterizing the dynamic colour change at colouration limits was not proved. The colour contrast of thermochromic systems was found to be directly related to the ratio of integrated intensity of lactone ring opened (solid) and lactone ring closed (liquid) carbonyl vibration characterized by infrared spectroscopy.
\end{abstract}

Keywords: reversible thermochromism, phase change, dynamic colour change, crystal violet lactone

\title{
1 Introduction
}

The reversible colour change of leuco dye based thermochromic (TC) systems is based on formation or destruction of a coloured complex between a $\mathrm{pH}$-sensitive colour former and a developer, driven by their interaction in an environment formed by a co-solvent $[1,2]$. A typical colour former in such systems is crystal violet lactone (CVL, Fig. 1). The ring opening of colourless CVL may be induced by an addition of a proton or through an increase in hydrogen bonding ability of the host environment, which is usually done by an addition of a developer. The coloured ring opened form can have a carbocationic or a three quinoid structure [3-5]. Phenolic compounds are often used as developers and alcohols, carboxylic acids, esters or ethers with long aliphatic chain are used as co-solvents [3, 6-12]. 

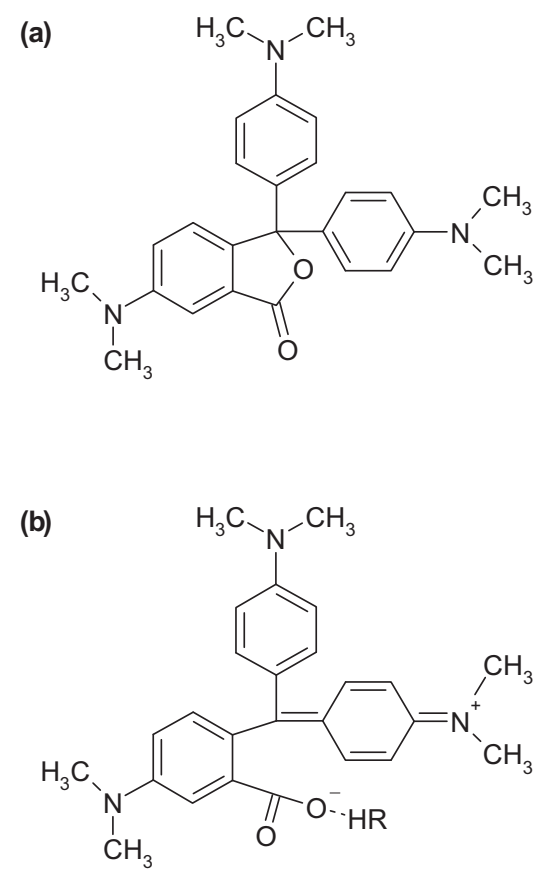

Fig. 1. Ring-closed colourless (a) and quinoid-immonium coloured (b) structure of CVL.

Thermochromic behaviour of three-component leuco dye based systems is influenced by a number of factors. One of the factors is the acidity of the developer and the acidity of the co-solvent; if the developer is a stronger acid, the system can exhibit only slight or no decolouration [8]. In addition, the steric factors of the colour former and developer are also influencing the formation of their complexes and thus the stoichiometry of colour complexes depends on the structure of both components $[11,13]$. The ring-closing mechanism is attributed to the solvation of the coloured complex of a colour former and a developer in melted co-solvent component [3,11]. A colour change of TC systems is closely related to their phase changes $[3,6,7]$, mainly influenced by the selected co-solvent. The structures of the co-solvent and developer influence the competitive binary interactions between the developer and colour former or co-solvent, respectively, and thus affect a colour contrast and decolourisation rates [6, 10, 14-16]. The correct balance of acidity of the developer and basicity of the colour former, balance in solubility and solvating powers of the developer and colour former in the co-solvent and interactions between the developer and co-solvent are important implications of the mechanism.

The limits of the composition range, which results in TC behaviour, can be found by complex experiments and depend on the chemical structure of components used in the formulation; in certain combinations, the systems can even exhibit colouration at molten state and decolouration in solid state $[7,9]$.

Long chain alcohols, predominantly used as co-solvents, show several phase transitions. On cooling, first a liquid-solid transition takes place and $\alpha$-phase [17] called also rotator phase [18-21] is formed. After the following solid-solid transition, a crystalline $\beta$-form or $\gamma$-form structures [17] are formed at low temperatures. A strong hysteresis can be observed for the solid-solid (rotator-crystalline) phase transition in comparison to the solid-liquid (rotator-liquid) phase transition [18, 20]. The liquid-solid and solid-solid transitions of even alcohols are clearly separated in DSC spectrum on cooling, whereas on heating they are overlapped [19,22]. 
In TC system employing the alcoholic co-solvent, the colouration was attributed by Tang et al. [7] to the formation of disordered rotator phase. Bourque [23] observed in mixtures decolouring upon heating that the substantial colour change is related to the formation or destruction of the low temperature rigid crystalline structure. Hajzeri et al. [10] demonstrated that decolouration of TC systems containing benzofluoran dye takes place much before solid-liquid transition, and connected the colouration (on cooling only) with the transition from the rotator phase to the rigid crystalline phase.

Opening of the lactone ring, thus formation of the colour complex, can be observed by infrared or Raman spectroscopy $[6,10,14,16,24]$. The formation of a coloured complex can by identified (i) by analyses of symmetric carbonyl $\mathrm{C}=\mathrm{O}$ vibration of lactone ring, which shifts to lower wavenumbers when the ring opens; (ii) by formation of peaks representing the symmetric and asymmetric carboxylate $\mathrm{COO}^{-}$stretching; and (iii) by double bond character of $\mathrm{C}-\mathrm{N}$ quinoid-immonium coloured structures. The optimal ratio of a colour former and a developer in binary mixtures $[10,24]$ was found to be not optimal in ternary TC systems $[6,10,14]$.

The optimal ratio of components was also observed by measuring the reflectance spectra of solid TC systems $[3,13,25]$. As we have shown in our previous papers [26, 27], the dynamic colour change can be described by several characteristics, and they vary depending on the concentration ratio of all three components. The work presented in this paper connects these colour characteristics with phase changes and changes in chemical structure related to the formation or destruction of the coloured complex in three-component TC systems.

\section{Materials}

All chemicals were purchased from Tokyo Chemical Industry and were used without further purification. Three-component TC systems containing crystal violet lactone (6-(dimethylamino)-3,3bis[p-(dimethylamino) phenyl] phthalide, $>95 \%$ ) as colour former, bisphenol A (BPA, 2,2-bis(4hydroxyphenyl)propane, $>99 \%$ ) as developer and 1-tetradecanol (TD, $>98 \%$ ) as the co-solvent were prepared within the scope of previously published paper [27]. Current paper focuses on further investigation of selected ternary TC systems and the corresponding binary mixtures with molar ratio of their components as given in Table 1.

Table 1 Molar ratios of components in ternary TC systems and binary TD-CVL and TD-BPA mixtures.

\begin{tabular}{|c|c|c|c|}
\hline \multicolumn{4}{|c|}{ Ternary TC systems $\left(x_{\mathrm{TD}} / x_{\mathrm{BPA}} / x_{\mathrm{CVL}}\right)$} \\
\hline $60 / 7 / 1$ & $80 / 7 / 1$ & $100 / 7 / 1$ & $120 / 7 / 1$ \\
\hline $60 / 4 / 1$ & $80 / 4 / 1$ & $100 / 4 / 1$ & $120 / 4 / 1$ \\
\hline $60 / 1 / 1$ & $80 / 1 / 1$ & $100 / 1 / 1$ & $120 / 1 / 1$ \\
\hline \multicolumn{4}{|c|}{ Binary TD-CVL mixtures $\left(x_{\mathrm{TD}} / x_{\mathrm{CVL}}\right)$} \\
\hline $60 / 1$ & $80 / 1$ & $100 / 1$ & $120 / 1$ \\
\hline \multicolumn{4}{|c|}{ Binary TD-BPA mixtures $\left(x_{\mathrm{TD}} / x_{\mathrm{BPA}}\right)$} \\
\hline $100 / 7$ & \multicolumn{2}{|c|}{$100 / 4$} & \\
\hline
\end{tabular}




\section{Methods}

Phase transitions of TC systems, binary mixtures and pure co-solvent were monitored by DSC analysis using Perkin Elmer Pyris 1 DSC device. The temperature range of $10-50{ }^{\circ} \mathrm{C}$ and the rate of temperature change of $2{ }^{\circ} \mathrm{C} \mathrm{min}^{-1}$ were set to be in match with the setup applied in measurements of the colour change [27]. For the evaluation, the second heating-cooling cycle was considered, to minimize the influence of sample preparation and its previous thermal history. The obtained temperature data in DSC measurements refer to the temperature of the reference due to the limiting heat transfer of the sensor.

Measurements of infrared spectra were done by FTIR spectrometer Bruker Vertex 80 using ATR technique with a diamond crystal. The objective was to measure TC systems in their coloured (solid) and decoloured (liquid) state. All samples of binary and ternary mixtures along with pure TD were measured at 20 and $50{ }^{\circ} \mathrm{C}$. First, a drop of liquefied sample was placed on top of the diamond crystal when the sample compartment was heated to higher temperature. Secondly, the sample was cooled down and the spectra at lower temperature were recorded. Then the sample compartment was heated to higher temperature again and the spectra were recorded. All spectra were recorded in the range of 4000-650 $\mathrm{cm}^{-1}$, with $4 \mathrm{~cm}^{-1}$ resolution and 64 scans. Recorded spectra were baseline corrected and normalized according to $\mathrm{CH}_{2}$ asymmetric stretching vibration of the aliphatic chain of the co-solvent at $2917 \mathrm{~cm}^{-1}$ in solid and at $2922 \mathrm{~cm}^{-1}$ in liquid state. Infrared spectra of pure CVL and BPA were measured at $20{ }^{\circ} \mathrm{C}$ by the same setup, only the powdered compounds were pressed toward the diamond crystal assuring good contact at the interface.

The results of DSC and FTIR analyses were compared with characteristics of the dynamic colour change - the colour contrast $C C_{\Delta E_{\mathrm{C}}}$ and temperatures characterizing the start and the end of colour changes, as obtained from the cumulative colour difference $\Delta E_{\mathrm{C}}$. Details on this evaluation can be found elsewhere [27]. The temperature $T_{\mathrm{DS}}$ (in [27] labelled as $T_{1}$ ) indicates the temperature at which the decolouration process starts at heating and $T_{\mathrm{DE}}\left(T_{2}\right.$ in [27]) indicates the temperature at which the decolouration ends. Analogous subscript convention applies for the temperatures $T_{\mathrm{CS}}$ and $T_{\mathrm{CE}}\left(T_{3}\right.$ and $T_{4}$ in [27], respectively) specified for the colouration process at cooling.

\section{Results}

\subsection{Phase transitions of binary and ternary mixtures}

The content of CVL in binary TD-CVL mixtures has almost no influence on their phase transitions in comparison to pure TD. As expected, there is only a single distinct peak on heating (see Fig. 2a). According to the literature [19,22], this peak represents the overlapping solid-solid and solid-liquid transitions. On cooling, firstly the liquid-solid transition occurs, forming a rotator phase, and by further cooling, the solid-solid transition takes place, forming a crystalline structure of the co-solvent. The melting point of pure TD co-solvent is $38.5^{\circ} \mathrm{C}$, and the liquid-solid and solid-solid transitions on cooling appear at 36.1 and $32.9^{\circ} \mathrm{C}$, respectively. The corresponding enthalpies $(H)$ are presented in Table 2. Although the liquid-solid and solid-solid transitions on cooling can be clearly resolved, for better comparison with the results for binary TD-BPA and ternary TC samples, the summed enthalpy of both liquid-solid and solid-solid transition is presented. The content of CVL in the analysed range has a negligible influence on the formation of TD rotator and crystalline structures. 

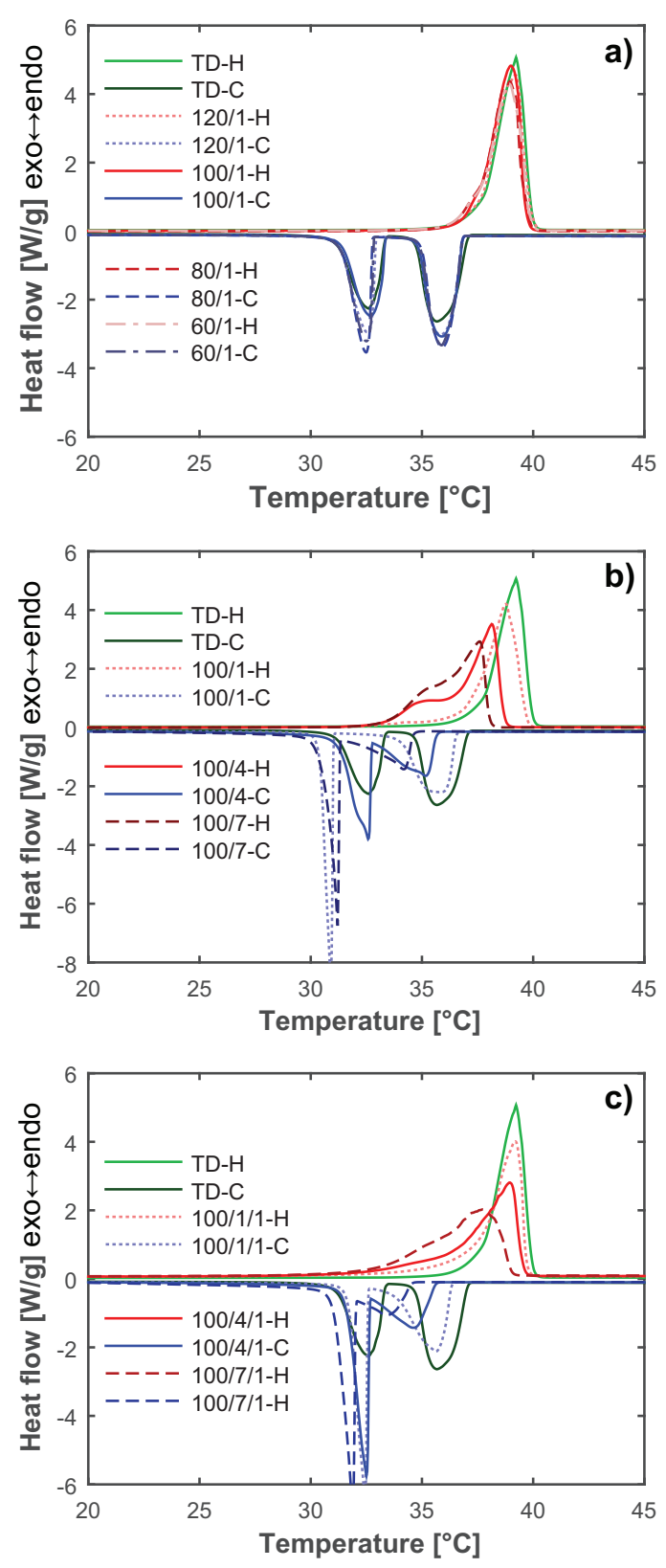

Fig. 2. DSC curves of binary TD-CVL (a), binary TD-BPA (b), and ternary TD-BPA-CVL mixtures (c) with given molar ratios, along with pure $\mathrm{TD}$, on heating $(\mathrm{H})$ and cooling $(\mathrm{C})$. 
Table 2 Enthalpies $\left[\mathrm{J} \cdot \mathrm{g}^{-1}\right]$ of pure TD and binary TD-CVL and TD-BPA mixtures.

\begin{tabular}{|c|cc||c|cc|}
\hline Pure TD & $\begin{array}{c}\text { Heating } \\
222,88\end{array}$ & $\begin{array}{c}\text { Cooling } \\
-220\end{array}$ & \multicolumn{3}{|c|}{ Binary TD-CVL $\boldsymbol{x}_{\mathbf{C V L}}=\mathbf{1}$} \\
\hline \hline \multicolumn{2}{|c|}{ Binary TD-BPA mixtures $\boldsymbol{x}_{\mathbf{T D}}=\mathbf{1 0 0}$} & $\boldsymbol{x}_{\mathbf{T D}}$ & Heating & Cooling \\
\hline $\boldsymbol{x}_{\text {BPA }}$ & Heating & Cooling & 60 & 219 & -217 \\
\hline 1 & 221 & -214 & 80 & 219 & -217 \\
4 & 217 & -193 & 100 & 223 & -221 \\
7 & 207 & -174 & 120 & 220 & -218 \\
\hline
\end{tabular}

Table 3 Enthalpies $\left[\mathrm{J} \cdot \mathrm{g}^{-1}\right]$ of ternary TC systems with varying $x_{\mathrm{TD}}$ and $x_{\mathrm{BPA}}$ and constant $x_{\mathrm{CVL}}=1$.

\begin{tabular}{|c|ccc||c|ccc|}
\hline \multicolumn{3}{|c||}{ Heating } & \multicolumn{4}{c|}{ Cooling } \\
\hline $\boldsymbol{x}_{\mathbf{T D} \downarrow / \boldsymbol{x}_{\mathbf{B P A}} \rightarrow}$ & 1 & 4 & 7 & $\boldsymbol{x}_{\mathbf{T D}} \downarrow / \boldsymbol{x}_{\mathbf{B P A}} \rightarrow$ & 1 & 4 & 7 \\
\hline 60 & 203 & 206 & 196 & 60 & -197 & -198 & -181 \\
80 & 209 & 209 & 204 & 80 & -205 & -201 & -192 \\
100 & 214 & 207 & 205 & 100 & -210 & -201 & -190 \\
120 & 220 & 212 & 210 & 120 & -217 & -207 & -201 \\
\hline
\end{tabular}

The addition of BPA into pure TD causes a shift of the melting point to lower temperatures and the broadening of the peak, i.e. the peaks corresponding to the solid-solid and solid-liquid transitions are less overlapped (see Fig. 2b). On cooling, the ratio of velocity of liquid-solid and solid-solid transition significantly changes. The increasing amount of BPA developer thus has a considerable influence on a formation of rotator and crystalline structure of TD co-solvent. As shown in Table 2, also the enthalpies of phase transitions significantly decrease with increasing amount of BPA, indicating lower crystallinity when compared to pure TD, what is in agreement with [10]. However, the results do not show evidence of the TD-BPA complex formation.

In ternary systems with $x_{\mathrm{TD}}=100$, the phase transitions follow the same tendencies as those in binary TD-BPA mixtures (see Fig. 2c). However, the rate of the absolute values of enthalpy decrease with increasing content of BPA is less distinct than in binary mixtures, especially for cooling, as shown in Table 3. It can lead to a conclusion that interactions of BPA with CVL in the coloured complex diminish the interaction of BPA with TD. This difference is in accordance with expectations of competitive interactions between the developer and colour former or co-solvent, respectively [10, 14].

In ternary $\mathrm{TC}$ systems, a new, almost negligible, transition at lower temperatures below the solidsolid transition can be observed. This transition could be identified in DSC curves for almost all ternary systems on heating by finding the onset temperature $\left(T_{\mathrm{OH} 1}\right)$ from the inflexion point of this transition, as given in Table 4. On cooling, it was possible to determine the endset temperature $\left(T_{\mathrm{EC} 3}\right)$ of this transition only for four samples with higher content of BPA in the system. It has to be noted that this variation of the heat flow from the baseline is generally low and that the determination of the onset (on heating) and endset (on cooling) of this transition might be with higher error than in case of other DSC characteristics. The start of the phase transition and start of the decolouration, specified by $T_{\mathrm{OH} 1}$ and $T_{\mathrm{DS}}$, respectively, seem to be related only in case of TC samples with the highest content of the developer, namely $x_{\mathrm{TD}} / x_{\mathrm{BPA}} / x_{\mathrm{CVL}}=60 / 7 / 1$ and $80 / 7 / 1$. The same applies for the relation between 
$T_{\mathrm{EC} 3}$ and $T_{\mathrm{CE}}$. In all other cases, the temperatures $T_{\mathrm{DS}}$ and $T_{\mathrm{CE}}$ are not close to any temperatures characterizing the phase change transition.

Table 4 Temperatures $\left[{ }^{\circ} \mathrm{C}\right]$ characterizing the phase change transitions and the dynamic colour change (grey cells); for the subscript convention please refer to Fig. 3.

\begin{tabular}{|c|cc|ccc|ccc|cc|}
\hline Sample & $\boldsymbol{T}_{\mathbf{O H} 1}$ & $\boldsymbol{T}_{\mathbf{D S}}$ & $\boldsymbol{T}_{\mathbf{S}-\mathbf{L}}$ & $\boldsymbol{T}_{\mathbf{D E}}$ & $\boldsymbol{T}_{\mathbf{E H} 1}$ & $\boldsymbol{T}_{\mathbf{O C} 1}$ & $\boldsymbol{T}_{\mathrm{CS}}$ & $\boldsymbol{T}_{\mathrm{L}-\mathbf{S}}$ & $\boldsymbol{T}_{\mathbf{E C} 3}$ & $\boldsymbol{T}_{\mathbf{C E}}$ \\
\hline $60 / 1 / 1$ & 30.0 & 11 & 38.8 & 39 & 39.4 & 36.0 & 35 & 35.4 & - & 10 \\
$60 / 4 / 1$ & 23.6 & 18 & 37.2 & 38 & 37.6 & 34.4 & 35 & 34.0 & 22.3 & 16 \\
$60 / 7 / 1$ & 23.7 & 22 & 36.4 & 38 & 37.3 & 32.9 & 35 & 32.1 & 20.3 & 21 \\
\hline $80 / 1 / 1$ & 29.4 & 11 & 39.1 & 39 & 39.7 & 36.3 & 35 & 35.6 & - & 10 \\
$80 / 4 / 1$ & 23.8 & 17 & 37.6 & 38 & 38.1 & 35.1 & 36 & 34.6 & 21.7 & 15 \\
$80 / 7 / 1$ & 23.3 & 22 & 36.8 & 38 & 37.2 & 33.7 & 36 & 33.2 & 21.5 & 19 \\
\hline $100 / 1 / 1$ & 29.3 & 11 & 39.3 & 39 & 39.7 & 36.3 & 35 & 35.6 & - & 10 \\
$100 / 4 / 1$ & 28.0 & 21 & 39.0 & 38 & 39.5 & 35.7 & 36 & 34.6 & - & 20 \\
$100 / 7 / 1$ & 28.8 & 22 & 37.7 & 38 & 39.0 & 34.5 & 36 & 33.4 & 18.6 & 20 \\
\hline $120 / 1 / 1$ & - & 20 & 38.8 & 38 & 39.6 & 36.4 & 35 & 35.1 & - & 18 \\
$120 / 4 / 1$ & 27.1 & 22 & 38.0 & 38 & 36.2 & 35.6 & 36 & 35.1 & - & 20 \\
$120 / 7 / 1$ & 27.2 & 22 & 37.4 & 38 & 38.0 & 34.8 & 36 & 34.3 & 14.8 & 20 \\
\hline
\end{tabular}

On the other hand, the temperatures characterizing the dynamic colour change at its decolouration limit can be related to the temperatures characterizing the phase change. As illustrated in Fig. 3 and Table 4, the temperature at which the decolouration ends $T_{\mathrm{DE}}$ is closely related to the melting of the system $\left(T_{\mathrm{S}-\mathrm{L}}\right)$ and endset of this transition $\left(T_{\mathrm{EH} 1}\right)$. On cooling, the $T_{\mathrm{CS}}$ is close to the temperatures characterizing the solidification of the sample $\left(T_{\mathrm{L}-\mathrm{S}}\right.$ and $\left.T_{\mathrm{OC} 1}\right)$. Considering the accuracy of measurement in case of the temperatures characterizing the colour change, it might be concluded that this relation is not dependent on the content of BPA developer in the system. It seems that the most rapid colouration and decolouration (see Fig. $3 \mathrm{~b}, \mathrm{c}$ ) is in samples with $x_{\mathrm{BPA}} \geq 4$ connected with rotatorsolid (endset temperature $T_{\mathrm{EC} 2}$ ) and solid-rotator (onset temperature $T_{\mathrm{OH} 2}$ ) transitions, respectively. The decolouration temperatures of TC systems containing CVL as the colour former are much more closely connected with phase changes than it was observed in systems containing benzofluoran dye [10]. 

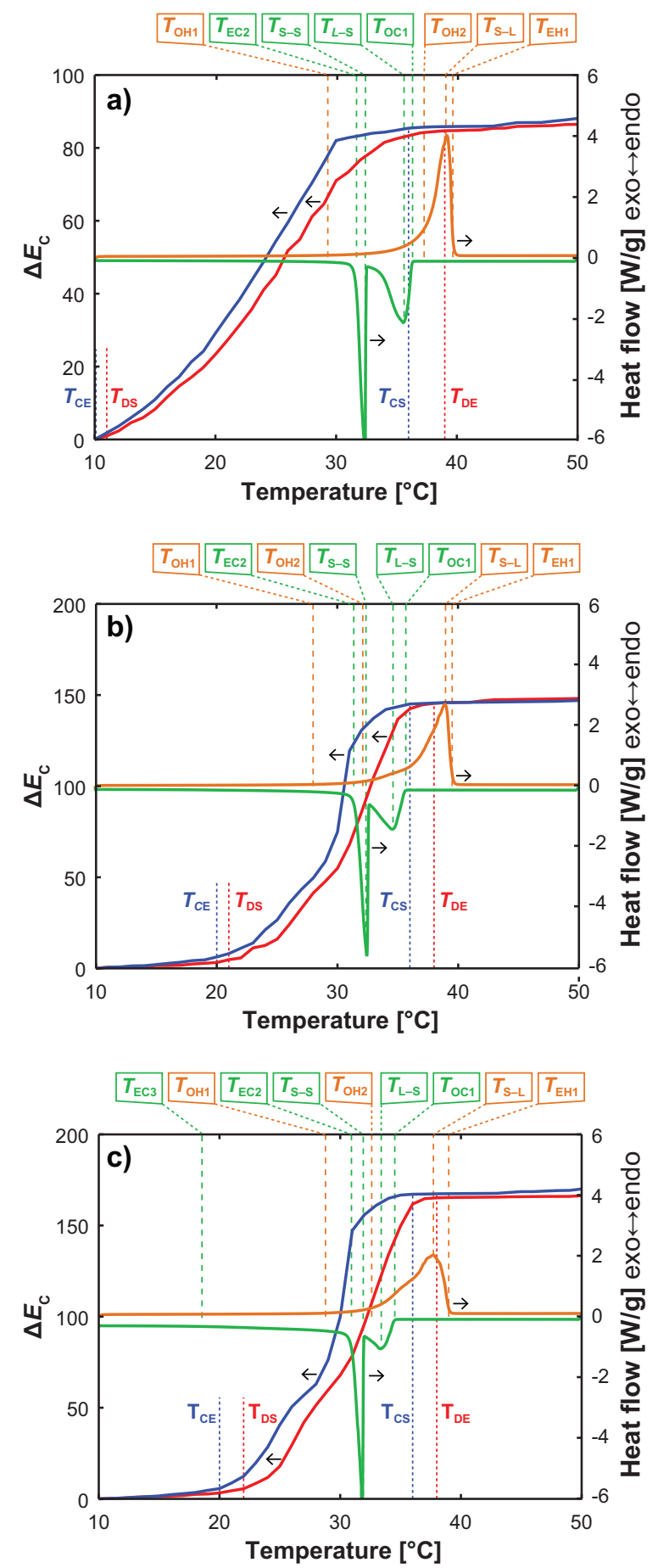

Fig. 3. DSC curves and the dynamic colour change described by the cumulative colour difference $\triangle \mathrm{EC}$ of ternary thermochromic systems with $x_{T \mathrm{D}} / x_{\mathrm{BPA}} / x_{\mathrm{CVL}}$ molar ratio equal to $100 / 1 / 1$ (a), 100/4/1 (b), and $100 / 7 / 1(c)$.

\section{2 Infrared spectroscopy}

The spectra of binary TD-BPA and TD-CVL mixtures along with the spectra of pure components are shown in Fig. 4 and Fig. 5, respectively. The main objective is the identification of characteristic peaks of pure components and their appearance or change in binary mixtures. In the solid state, the pure TD exhibits a broad peak with a maximum at $3273 \mathrm{~cm}^{-1}$, which is associated with the $\mathrm{OH}$ stretching 
vibration [28]. The single band character in this part of TD spectrum is referred to its $\beta$-form crystals [17]. The $\mathrm{OH}$ stretching of pure BPA has a maximum at $3320 \mathrm{~cm}^{-1}$. The binary TD-BPA mixtures have almost identical intensities and character of the $\mathrm{OH}$ stretching peak as a pure co-solvent. In molten state, TD co-solvent exhibits a single band peak with a maximum at about $3333 \mathrm{~cm}^{-1}$. Again, the bands in binary TD-BPA mixtures are almost identical to those belonging to pure TD. The $\mathrm{OH}$ stretching at high wavenumbers thus exhibits in binary mixtures as well as in ternary TC systems (not shown here) almost negligible variations comparing to pure TD co-solvent and they are considered insignificant regarding the difference in the vibration energy and integrated intensity. However, it should be pointed out that the broad peaks such as the described ones are not very selective to position and intensity because of the spectral background and low intensities.

a)

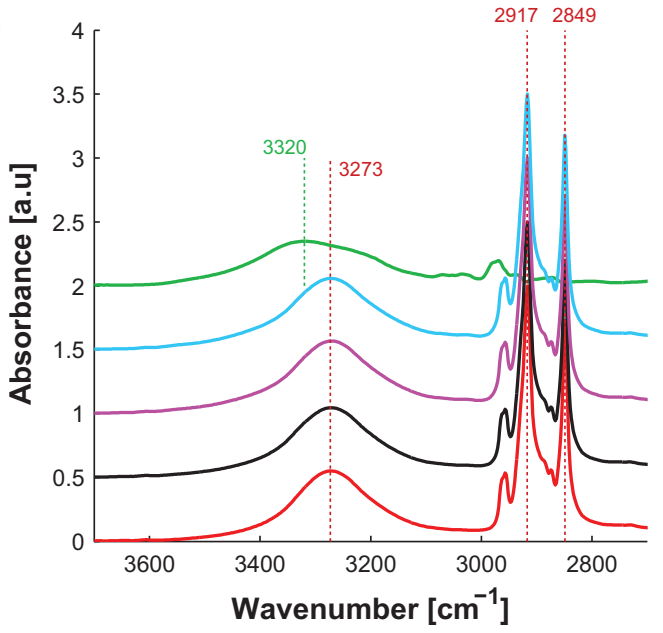

c)

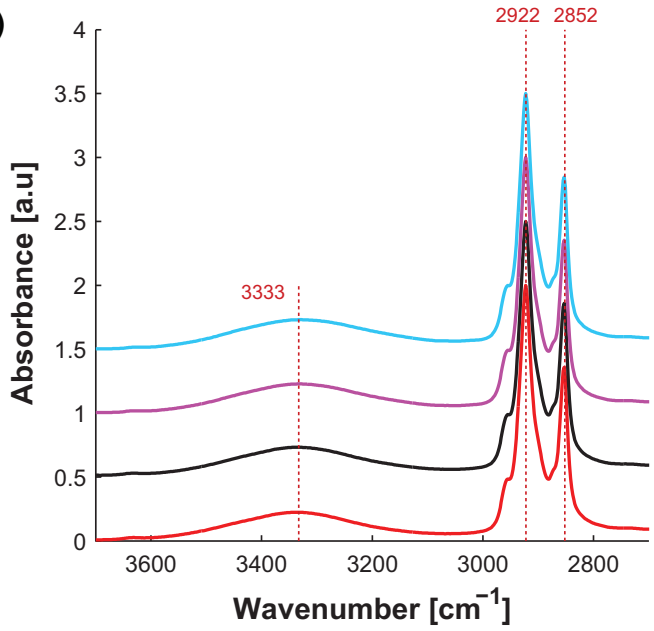

b)

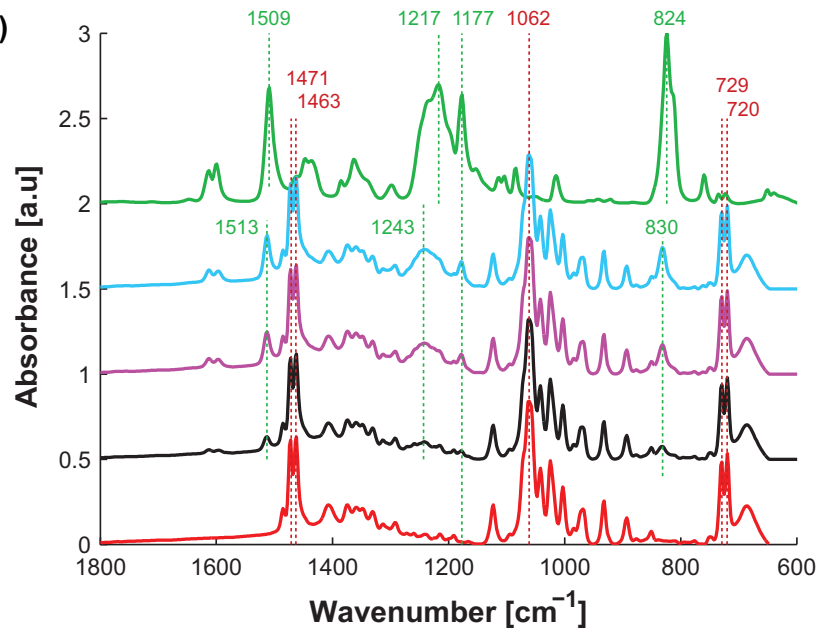

d)

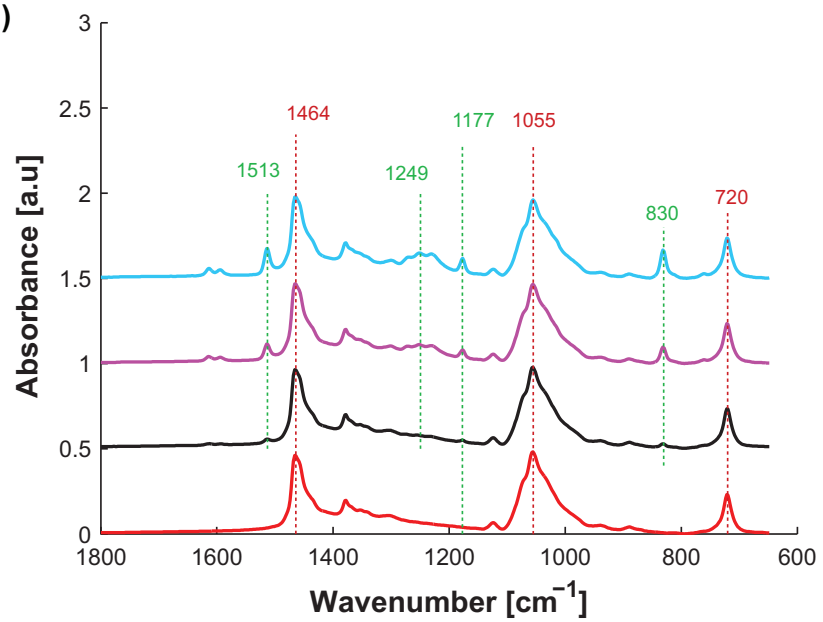

$100 / 7$

Fig. 4. Infrared spectra of binary TD-BPA mixtures with given molar ratios and pure components measured at $20{ }^{\circ} \mathrm{C}(\mathrm{a}, \mathrm{b})$ and $50{ }^{\circ} \mathrm{C}(\mathrm{c}, \mathrm{d})$. For better clarity, two selected spectral regions are presented and the spectra are vertically displaced. 

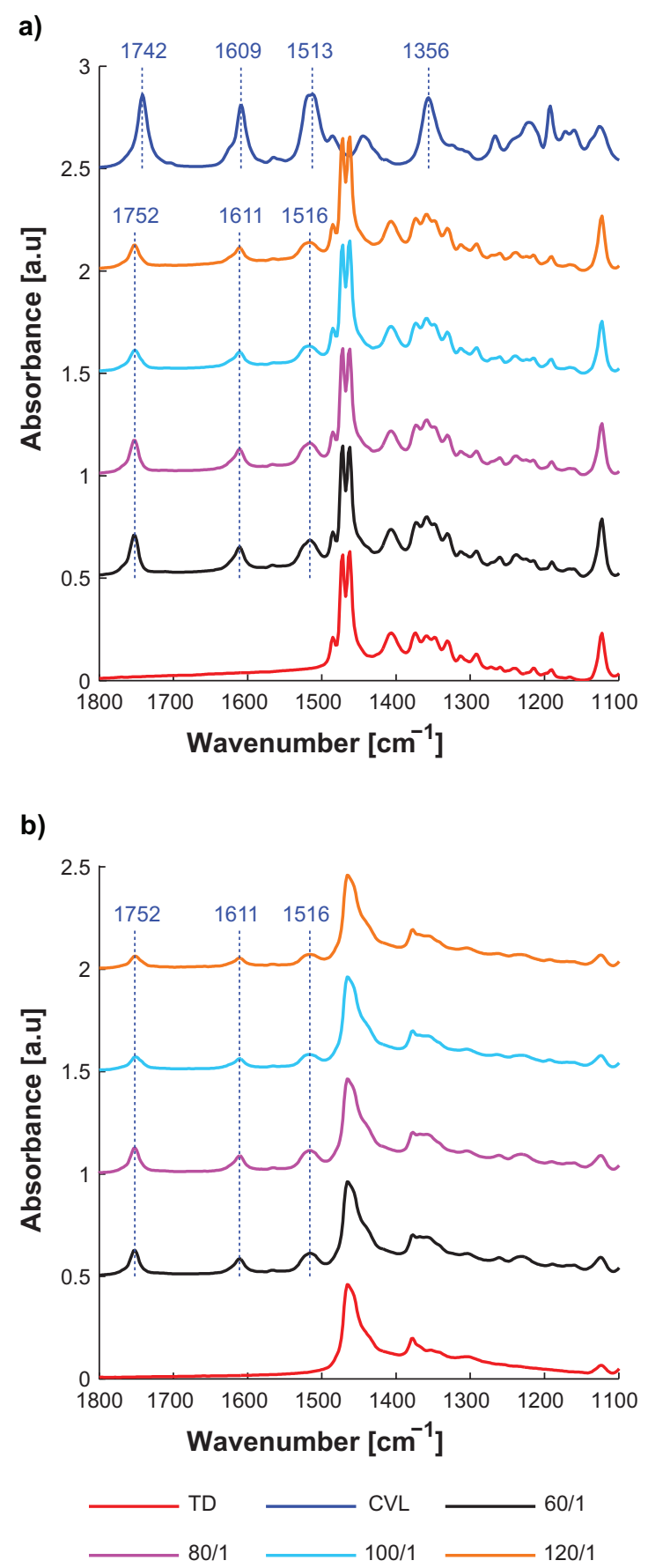

Fig. 5. Infrared spectra of binary TD-CVL mixtures with given molar ratios and pure components measured at $20^{\circ} \mathrm{C}$ (a) and $50{ }^{\circ} \mathrm{C}$ (b). For better clarity, the spectra are vertically displaced.

The presence of BPA in binary mixtures can be observed by a peak appearing at $1513 \mathrm{~cm}^{-1}$ (at 1509 $\mathrm{cm}^{-1}$ in pure BPA) both in solid and liquid state. It is associated with the $\mathrm{C}-\mathrm{C}$ stretching in the aromatic ring of BPA. The out-of-plane $\mathrm{C}-\mathrm{H}$ wag deformation in $p$-substituted aromatic ring is associated with the peak at $824 \mathrm{~cm}^{-1}$ in pure BPA and the peak at $830 \mathrm{~cm}^{-1}$ in binary mixtures [28]. The broad peak at $1217 \mathrm{~cm}^{-1}$ appearing in pure BPA is associated with phenol $\mathrm{OH}$ deformation and the peak at $1177 \mathrm{~cm}^{-1}$ is associated with the $\mathrm{C}-\mathrm{O}$ stretching and they are expected to interact [28]. The redistribution of the phenol $\mathrm{OH}$ deformation broad band in binary TD-BPA mixtures, with a maximum at $1243 \mathrm{~cm}^{-1}$ in solid and $1249 \mathrm{~cm}^{-1}$ in liquid, might be associated with less H-bonding interaction between BPA molecules due to the presence of the co-solvent. The peaks appearing at 
1471 and $1463 \mathrm{~cm}^{-1}$ are associated with TD co-solvent $\mathrm{CH}_{2}$ deformation and transform into a single band at $1464 \mathrm{~cm}^{-1}$ in molten state $[17,28]$. Similarly, the rocking doublet at 729 and $720 \mathrm{~cm}^{-1}$ in solid is replaced by a single band at $720 \mathrm{~cm}^{-1}$ in molten state [17]. The strong peak appearing at $1062 \mathrm{~cm}^{-1}$ in solid, attributed to the $\mathrm{C}-\mathrm{O}$ stretching, is overlapping with bands of the $\mathrm{CH}_{2}$ skeletal vibration [17]. In molten state, these bands blend into a one broad band with a maximum at $1055 \mathrm{~cm}^{-1}$. In case of binary mixtures, neither the intensity nor the distribution of these frequencies differs from those observed for pure TD; the same applies for ternary TC systems (not shown here). The results presented above imply that there are no or only negligible interactions between BPA developer and TD co-solvent. The coloured complex is then expected not to compete with other complex formation as it was reported for systems applying gallates as developers $[6,14]$.

As illustrated in Fig. 5, the presence of CVL can be detected in binary mixtures by three significant peaks. In pure CVL, the closed lactone ring carbonyl vibration is represented by the peak at $1742 \mathrm{~cm}^{-1}$ and the peaks appearing at 1609 and $1513 \mathrm{~cm}^{-1}$ represent the $\mathrm{C}-\mathrm{C}$ stretching of CVL aromatic rings. . All three peaks are slightly shifted in both solid and liquid binary TD-CVL mixtures to $1752 \mathrm{~cm}^{-1}$, 1611 and $1516 \mathrm{~cm}^{-1}$, respectively, and their intensities increase proportionally to the concentration of $\mathrm{CVL}$ in the mixture. The stretching of the $\mathrm{C}-\mathrm{N}$ bond at $1356 \mathrm{~cm}^{-1}$ in pure $\mathrm{CVL}$ is in binary mixtures overlapped with the $\mathrm{CH}_{2}$ deformations of the co-solvent and its intensity is not strong. Therefore, the evaluation of this CVL peak is limited in both binary and ternary mixtures. In the low-wavenumber region $\left(1100-650 \mathrm{~cm}^{-1}\right)$, the presence of CVL in binary mixtures and the formation of a coloured complex in ternary TC systems were not reflected by any vibration band.

Infrared spectra of ternary TC systems with the variation of BPA content are shown in Fig. 6. The formation of a coloured complex is connected with the opening of the lactone ring of CVL. In solid TC systems, the carbonyl vibration of a closed lactone ring shifts to the lower frequency of symmetric vibration of ring-opened $\mathrm{H}$-bonded $\mathrm{C}=\mathrm{O}$ in carboxylate group [10, 16, 24, 28]. In measured spectra, these peaks appear at 1752 and $1726 \mathrm{~cm}^{-1}$, respectively. In molten state, the carbonyl vibration is present at $1744 \mathrm{~cm}^{-1}$. The changes in carbonyl vibrations are discussed in the following text. The opening of the ring is also indicated by the two newly appearing peaks representing the formation of $\mathrm{COO}^{-}$group $[10,24,28]$. The peaks at 1584 and $1360 \mathrm{~cm}^{-1}$ are attributed to the asymmetric and symmetric carboxylate stretching, respectively and shift to 1595 and $1358 \mathrm{~cm}^{-1}$ in molten state. MacLaren and White [24] assigned the vibration at $1584 \mathrm{~cm}^{-1}$ to the asymmetric vibration of $\mathrm{COO}^{-}$ and also to the $\mathrm{C}-\mathrm{N}$ vibration with a significant double bond character. In TC systems using benzofluoran dye as a colour former [10], the carboxylate and quinoid-iminium form in the coloured complex could be distinguished from each other. In case of CVL in our TC systems, they might be overlapping in the region. Concerning the intensities of the symmetric $\mathrm{COO}^{-}$vibrations, the main contribution to the $1584 \mathrm{~cm}^{-1}$ vibration is expected to be mainly due to the asymmetric carboxylate vibration. Anyhow, the peak represents the lactone ring opening and formation of coloured species. 

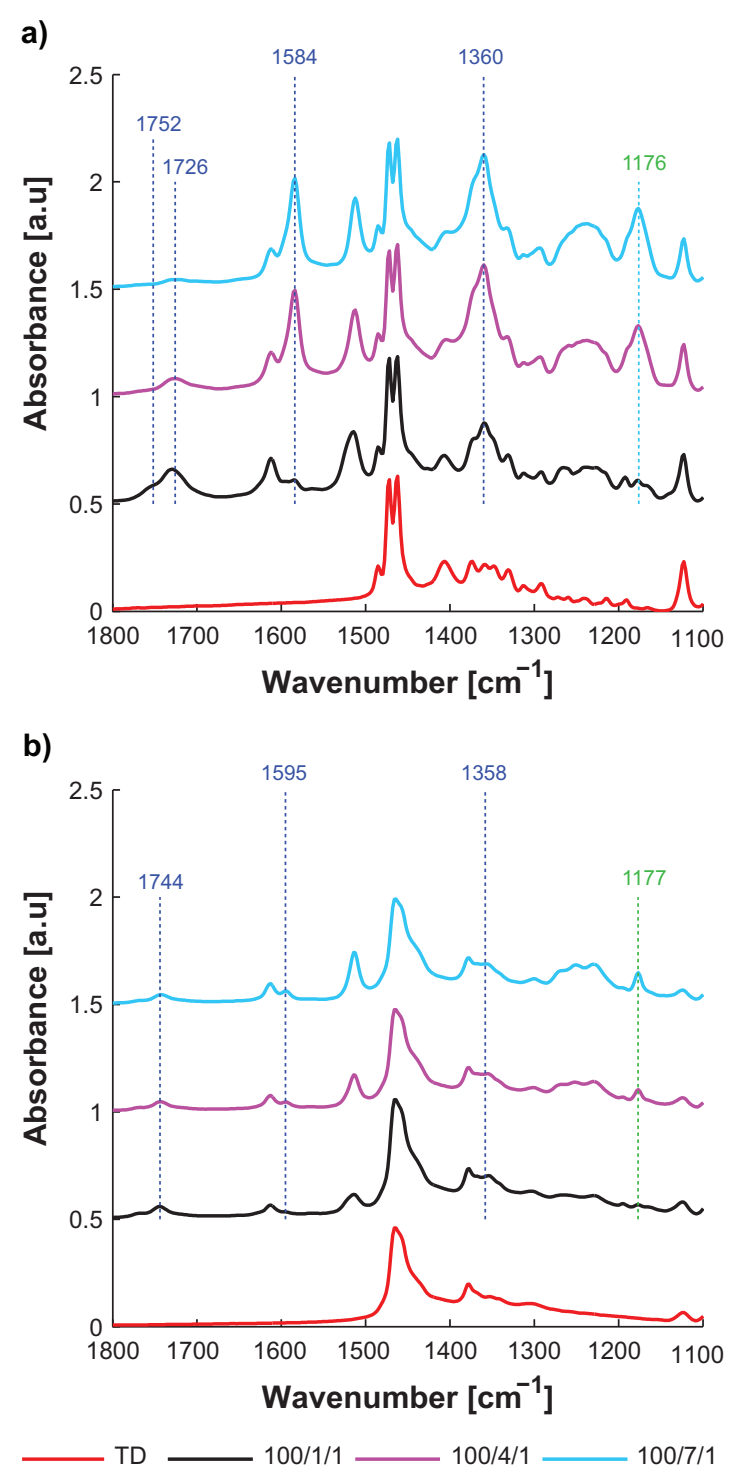

Fig. 6. Infrared spectra of ternary TC systems with given molar ratios and pure TD measured at $20{ }^{\circ} \mathrm{C}$ (a) and $50{ }^{\circ} \mathrm{C}$ (b). For better clarity, the spectra are vertically displaced.

When the molar ratio of $x_{\mathrm{BPA}} / x_{\mathrm{CVL}}$ is equal to $1 / 1$, there is still apparent relatively strong vibration of lactone $\mathrm{C}=\mathrm{O}$ stretching at $1752 \mathrm{~cm}^{-1}$ in solid samples (see Fig. 6a). The asymmetric carboxylate vibration is almost negligible and the symmetric one is slightly stronger at $x_{\mathrm{BPA}} / x_{\mathrm{CVL}}$ equal to $1 / 1$. When the $x_{\mathrm{BPA}} / x_{\mathrm{CVL}}$ is equal to $4 / 1$ and $7 / 1$, both peaks of carboxylate vibrations remain almost the same in the integrated intensity for all concentrations of TD. However, the integrated intensity of $\mathrm{C}=\mathrm{O}$ vibration of peak at $1726 \mathrm{~cm}^{-1}$ rapidly decreases with the content of BPA (see Fig. 6a), as was also observed in [16]. The disappearing of the peak might be connected with further strengthened $\mathrm{H}$ bonding of carbonyl group by BPA molecules. The stronger electron withdrawing power caused by the developer seems to diminish the $\mathrm{C}=\mathrm{O}$ double bond character. The carbonyl vibration of ternary $\mathrm{TC}$ systems in molten state appears at $1744 \mathrm{~cm}^{-1}$ (see Fig. 6b), which is lower than the vibration of the same group in binary TD-CVL mixtures at $1752 \mathrm{~cm}^{-1}$ (see Fig. 5b). This may be considered as an indication of H-bonding interactions between CVL and BPA molecules also in the molten state of ternary TC systems that do not necessarily lead to lactone ring opening. Also a small shoulder at 1595 $\mathrm{cm}^{-1}$ representing the lactone ring open $\mathrm{COO}^{-}$can be observed in almost all ternary TC systems in 
molten state and rises with the content of BPA in the system. This means that the coloured complex is not fully destroyed. The colour analyses have shown [27] that the path of colour change in CIELAB colour space starts and ends at different points, depending on the $x_{\mathrm{TD}} / x_{\mathrm{BPA}} / x_{\mathrm{CVL}}$ molar ratio. The length of this path also varies, and it is represented by the colour contrast $C C_{\Delta E_{\mathrm{C}}}$. The colour contrast characterises the dynamic colour change in terms of perceived difference between coloured and decoloured state. In IR spectra, the colouration and decolouration of the system is identified by presence or absence of characteristic vibrations of colour complex. If we divide the integrated intensity of characteristic vibration of the coloured (solid) and decoloured (liquid) TC system, a measure describing the contrast (differences) in structure is obtained and will be in further text marked as $R I I$ (relative integrated intensity). We expect that the perceived colour contrast represented by $C C_{\triangle E_{\mathrm{C}}}$ should be related to the contrast in structure represented by RII.

First, the integrated intensities of the symmetric carbonyl $\mathrm{C}=\mathrm{O}$ vibration and the asymmetric and symmetric $\mathrm{COO}^{-}$stretching for solid as well as liquid state of all ternary TC systems were computed. For each sample, RII of specific vibration in solid and liquid state was obtained. The comparison between this ratio and the colour contrast was based on the determination of sample Pearson linear correlation coefficient $R$ between these two parameters. The largest correlation of $R I I$ and $C C_{\triangle E_{\mathrm{C}}}$, reflected in correlation coefficient about -0.96 , was found in case of the $\mathrm{C}=\mathrm{O}$ vibration (see Fig. 7). The correlation is negative due to the disappearing of the $\mathrm{C}=\mathrm{O}$ vibration in $\mathrm{TC}$ systems with increasing $x_{\mathrm{BPA}}$. The correlation coefficient of the colour contrast and the ratio of integrated intensities of the symmetric $\mathrm{COO}^{-}$stretching is below 0.7 and for the asymmetric $\mathrm{COO}^{-}$stretching it is even less than 0.5. This indicates that it is the vibration of carbonyl group of lactone closed and open ring what is strongly connected with the colour contrast of studied ternary TC systems.

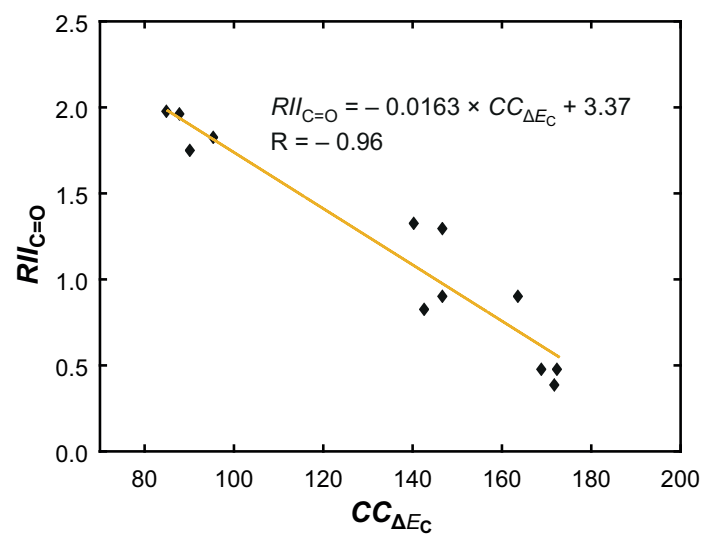

Fig. 7. Correlation between the relative integrated intensity $R I I$ of lactone $\mathrm{C}=\mathrm{O}$ vibration and colour contrast $C C_{\Delta E_{\mathrm{C}}}$ of all TC systems presented in Table 1.

\section{Conclusions}

Ternary thermochromic systems containing crystal violet lactone (colour former), bisphenol A (developer) and 1-tetradecanol (co-solvent) were analysed in terms of phase transitions and changes in characteristic group vibrations in infrared spectra. In addition, the results were compared with the data obtained for binary TD-CVL and TD-BPA mixtures, as well as for pure components. The content of 
the colour former in observed concentration range did not have any influence on phase changes in binary TD-CVL mixtures. Observed differences in phase changes of presented model systems analysed by DSC are mainly influenced by BPA developer, and this influence is less apparent in ternary thermochromic systems when compared to the binary TD-BPA mixtures with the same $x_{\mathrm{TD}} / x_{\mathrm{BPA}}$ molar ratio. The temperature at which the decolouration stops was closely related to the melting point and the end of the solid-liquid transition. On cooling, the temperature at which the colouration starts was close to the temperature characterizing the start of liquid-solid transition. However, the temperatures characterizing the dynamic colour change at colouration limits were in most cases far from the temperatures characterizing the liquid-solid and solid-solid phase transitions. The possible connection with a weak shoulder observed in DSC data at the temperatures below the solid-solid transition has not been proved within the presented study. Nevertheless, both the onset and endset temperatures of this indistinctive transition are close to the temperatures characterizing the dynamic colour change at colouration limits in case of two samples with the high content of BPA, namely with $x_{\mathrm{TD}} / x_{\mathrm{BPA}} / x_{\mathrm{CVL}}$ equal to $60 / 7 / 1$ and $80 / 7 / 1$.

The connection of the dynamic colour change with vibrational analyses was accomplished through the correlation of the colour contrast with the contrast in the structure represented by the ratio of integrated intensities of characteristic groups of CVL lactone closed and opened ring. This analysis revealed that the colour contrast is only weakly linked to the ratio of integrated intensities of the symmetric and asymmetric lactone ring opened carboxylate vibrations. On the other hand, a very strong correlation (more than -0.95) with the ratio of integrated intensities was observed in case of symmetric carbonyl vibration. The decrease in the ratio of integrated intensities is mainly due to decreasing intensity of the carbonyl vibration in solid samples, caused by a large content of the developer. Therefore, the lactone ring carbonyl vibration is proposed as a suitable indicator of the colour contrast characterizing the dynamic colour change of model systems presented in this study.

\section{Acknowledgements}

This work was supported by the Ministry of education, youth and sports of the Czech Republic, through project LD14098 and Slovenian Research Agency (project no. L2-5571) and En-Fist centre of excellence. The authors acknowledge Roman Svoboda from the University of Pardubice for consultations regarding the DSC data evaluation.

\section{References}

[1] White MA, LeBlanc M. Thermochromism in commercial products. J Chem Educ 1999;76(9):1201-5; DOI:10.1021/ed076p1201

[2] Seeboth A, Lötzsch D. Thermochromic and Thermotropic Materials. Singapore: Pan Stanford; 2014; DOI: I10.4032/9789814411035

[3] Burkinshaw S, Griffiths J, Towns A. Reversibly thermochromic systems based on pH-sensitive spirolactone-derived functional dyes. J Mater Chem 1998;8(12):2677-83; DOI: 10.1039/a805994b 
[4] Kuzuya M, Usui T, Ito S, Miyake F, Nozawa S, Okuda T. Substituent Effects and Structural Limitations in the Conversion of 3,3-Diarylphthalides to 4,4-Diaryl-3,4-dihydro-1(2H)phthalazinones. Chem Pharm Bull 1980;28(12):3561-9; DOI: 10.1248/cpb.28.3561

[5] Takaoka K, Maeda S, Miura H, Endo K, Chong DP. Theoretical valence XPS and UV-visible absorption spectra of four leucodyes using MO calculations. Bull Chem Soc Jpn 1998;71:807-16.

[6] MacLaren D, White MA. Design rules for reversible thermochromic mixtures. J Mater Sci 2005;40(3):669-76; DOI: 10.1007/s10853-005-6305-x

[7] Tang H, MacLaren DC, White MA. New insights concerning the mechanism of reversible thermochromic mixtures. Can J Chem 2010;88(11):1063-70; DOI: 10.1139/V10-069

[8] Zhu C, Wu A. Studies on the synthesis and thermochromic properties of crystal violet lactone and its reversible thermochromic complexes. Thermochim Acta 2005;425(1-2):7-12; DOI: 10.1016/j.tca.2003.08.001

[9] Bourque AN, White MA. Control of thermochromic behaviour in crystal violet lactone (CVL)/alkyl gallate/alcohol ternary mixtures. Canad J Chem 2015;93(1):22-31; DOI: 10.1139/cjc2014-0251

[10] Hajzeri M, Bašnec K, Bele M, Klanjšek Gunde M. Influence of developer on structural, optical and thermal properties of a benzofluoran-based thermochromic composite. Dyes Pigm 2015;113(0):754-62; DOI: 10.1016/j.dyepig.2014.10.014

[11] Seeboth A, Lötzsch D, Potechius E, Vetter R. Thermochromic effects of leuco dyes studied in polypropylene. Chin J Polym Sci 2006;24(4):363-8; DOI: 10.1142/S0256767906001400

[12] Katsuyuki F, Kuniyuki S. The Plilot Ink Co., Ltd, assignee. Thermochromic microencapsulated pigments. US6494950 B1. 2002 11/17.

[13] Luthern J, Peredes A. Determination of the stoichiometry of a thermochromic color complex via Job's method. J Mater Sci Lett 2000;19(3):185-8, DOI: 10.1023/A:1006790104175

[14] MacLaren D, White MA. Competition between dye-developer and solvent-developer interactions in a reversible thermochromic system. J Mater Chem 2003;13(7):1701-4; DOI: 10.1039/b302250a

[15] Sekiguchi Y, Takayama S, Gotanda T, Sano K. Molecular Structures of the Coloring Species of a Leuco Dye with Phenolic Color Developers. Chem Lett 2007;36(8): 1010-1; DOI:

10.1246/cl.2007.1010

[16] Raditoiu V, Raditoiu A, Wagner L, Amariutei V, Nicolae CA. Thermochromic Systems based on Complexes of Some Triarylmethane Dyes. Rev Chim 2013;64(2)2013:147-51

[17] Tasumi M, Shimanouchi T, Watanabe1 A, Goto R. Infrared Spectra of Normal Higher alcohols-I. Spectrochim Acta 1964;20(4):629-66; DOI: 10.1016/0371-1951(64)80060-6

[18] Sirota EB, Wu XZ. The rotator phases of neat and hydrated 1-alcohols. J Chem Phys 1996;105(17):7763-73; DOI: 10.1063/1.472559

[19] Ventola L, Ramirez M, Calvet T, Solans X, Cuevas-Diarte MA, Negrier P, Mondieig D, van Miltenburg JC, Oonk HAJ. Polymorphism of N-Alkanols: 1-Heptadecanol, 1-Octadecanol, 1Nonadecanol, and 1-Eicosanol. Chem of Mater 2002;14(2):508-17; DOI: 10.1021/cm011010h 
[20] Ventola L, Calvet T, Cuevas-Diarte MA, Solans X, Mondieig D, Negrier P, van Miltenburg JC. Solid State Equilibrium in the N-Alkanols Family: The Stability of Binary Mixed Samples. Phys Chem Chem Phys 2003;5(5):947-52; DOI: 10.1039/b210333h

[21] Ventola L, Calvet T, Cuevas-Diarte MA, Oonk HAJ, Mondieig D. Solid-Solid and Solid-Liquid Equilibria in the N-Alkanols Family: C18H37OH-C20H41OH System. Phys Chem Chem Phys 2004;6(13):3726-31; DOI: 10.1039/b402073a

[22] van Miltenburg JC, Oonk, HAJ, Ventola L. Heat Capacities and Derived Thermodynamic Functions of 1-Octadecanol, 1-Nonadecanol, 1-Eicosanol, and 1-Docosanol between $10 \mathrm{~K}$ and $370 \mathrm{~K}$. J Chem Eng Data 2001;46(1):90-7; DOI: 10.1021/je000048s

[23] Bourque A. Investigations of reversible thermochromism in three-component systems; Dalhousie University; 2014.

[24] MacLaren D, White MA. Dye-developer interactions in the crystal violet lactone-lauryl gallate binary system: implications for thermochromism. J Mater Chem 2003;13(7):1695-700; DOI: $10.1039 / \mathrm{b} 302249 \mathrm{~h}$

[25] Luthern J, Peredes A. Determination of the stoichiometry of a thermochromic color complex via the method of continuous variation. J Mater Sci Lett 2003;22(12):881-4; DOI: 10.1023/A:1024410703372

[26] Panák O, Hauptman N, Klanjšek Gunde M, Kaplanová M. Colorimetric characterisation of thermochromic composites with different molar ratios of components. J Print Media Technol Res 2012;1(2):113-20; DOI: 10.14622/JPMTR-1204

[27] Panák O, Držková M, Kaplanová M. Insight into the evaluation of colour changes of leuco dye based thermochromic systems as a function of temperature. Dyes Pigm 2015;120(0):279-287; DOI: 10.1016/j.dyepig.2015.04.022

[28] Colthup NB. Daly LH. Wiberley SE, Introduction to Infrared and Raman Spectroscopy, 3rd ed. Academic press; 1990; ISBN: 978-0-12-182554-6 